\title{
Exact solution for the massless Cylindrically Symmetric Scalar Field in General Relativity, with Cosmological Constant
}

\author{
D.Momeni将 \\ Department of Physics,Azad University, P.O. Box 31485-313, Karaj, Iran \\ H. Miraghaeit \\ Department of Physics, Sharif University of Technology, P.O. Box 113659161, Tehran, Iran \\ In this article we present a new exact solution for scalar field with cosmological \\ constant in cylindrical symmetry. Associated cosmological models is discussed. One \\ of them describes a Cyclic universe. \\ PACS numbers: 04.30.-w, 96.10.+i, 11.25.-w
}

\section{INTRODUCTION}

In 1959 Buchdahl introduced a new generating method based on an old fascinated method which arisen from potential theory (reciprocal transformation) for generating exact solution from a known perfect fluid solution to another one [1]. It's family divided into two subclass which in one, scalar field depends only on radial coordinates (Cylindrical or Spherical ) and in the other -as Wyman mentioned- scalar fields depend only on time [2]. For a gravity which is coupled to a neutral, massless scalar field, Wyman suggested a method of solution in power series valid for a scalar field depends only on time. Wyman's static spacetime with a non static scalar field could not integrated explicitly as we noted it in [3]. Another generalizations of this family to higher dimensional cases could be found in [4]. Recently Vuille found a new exact solution for a massless scalar field with cosmological constant in plane symmetry [6]. Late Anzhong Wang studied cylindrically symmetric spacetimes with homothetic self-similarity in the context of Einstein's Theory of Gravity [9]. Also Sarioglu and Tekin generalize the four dimensional cylindrically symmetric static spacetimes with a negative cosmological constant to higher dimensions[17]. There is no general solution for Buchdahl massless scalar field which is minimally coupled to a massive one or in the presence of cosmological constant. In this letter ,First we present a new cylindrically symmetric solution for a massless scalar field coupled minimally to gravity but contains cosmological constant. This exact solution is regular for all values of scalar field parameter $\xi^{2}>0$. The purely complex value of $\xi$ contradicts with our assumption that the scalar field function must be real.But this complex value for $\xi$ imposes a naked singularity. Then we show this solution represents Buchdahl solution in the absence of the cosmological constant and reduces to Levi-Civita- $\Lambda$ solution $(L C \Lambda)$ when the scalar field set to be zero.Thus we generalized both Buchdahl and $L C \Lambda$ solutions and we solved an old obscure problem since 1959.Also we describe their cosmological metrics and show that there are two different family of models :one which is simpler corresponds to an inflationary model that the scalar field has the role of inflaton. The Hubble parameter of this model remains constant. Hence this model has

\footnotetext{
* Corresponding author
} 
no time evolution. This solution matches a negative cosmological constant which is harmful for accelerating expansion of universe in now era.Another solution describes a FriedmannRobertson-Walker universe with a period of about cosmic time scale.This solution related to a Cyclic model which has a significant braneworld scenario of the universe.

\section{FIELD EQUATIONS}

We begin with a general cylindrically symmetric metric in Weyl coordinates $(t, r, \varphi, z),{ }^{1}$

$$
d s^{2}=-e^{u(r)} d t^{2}+d r^{2}+e^{v(r)} d \varphi^{2}+e^{w(r)} d z^{2}
$$

Field equation for a massless minimally coupled scalar field in the presence of a cosmological constant term $\Lambda$ is reading as:

$$
R_{\mu \nu}-\Lambda g_{\mu \nu}=\phi_{; \mu} \phi_{; \nu}
$$

We labled metric functions as $u_{i}=\{u(r), v(r), w(r)\}, \phi \equiv \phi(r), \dot{f}=\frac{d f}{d r}$. In terms of these functions we can rewrite field equation (2) in the following succinct forms:

$$
\begin{array}{r}
2 u_{i}^{\prime \prime}+u_{i}^{\prime} \sum_{j=1}^{3} u_{j}^{\prime}-4 \Lambda=0, i=\{1,2,3\} \\
2 \sum_{j=1}^{3} u_{j}^{\prime \prime}+\sum_{j=1}^{3} u_{j}^{\prime 2}-4 \Lambda=4 \phi^{\prime 2}
\end{array}
$$

This system of differential equations has no simple solution and only could be reduced to a set of non linear Sturm-Liouvile equations.

\section{EXACT SOLUTIONS}

In this section we present some different possible solutions of $(3,4)$.

Case (1) : solution with $u(r)=$ constant, $w(r)=$ constant, $\phi^{\prime}=0$

We prove that if we have $u(r), w(r)=$ constant then we deduce flat space. Starting from the equation (3) for $i=1,3$ we get $\Lambda=0$ and by comparing it with (4) we observe that $\phi=c$ (a constant) which we can take it zero.We have:

$$
2 v^{\prime \prime}+v^{2}=0
$$

The exact solution for this odinary differential equation is²:

$$
v(r)=2 \log (a r+b)
$$

\footnotetext{
${ }^{1}$ I will mostly use natural units $\hbar=c=1$ and $8 \pi G=1$.

${ }^{2} \log (x)=\int_{1}^{x} \frac{d \zeta}{\zeta}$
} 
The constant $b$ may be turned to zero by changing scales along the $t$ and $z$ axes and choosing the zero point of the $r$ coordinate. Then the line element (1) transformed to;

$$
d s^{2}=-d t^{2}+d r^{2}+(a r)^{2} d \varphi^{2}+d z^{2}
$$

By applying change of coordinates:

$$
\tilde{\varphi}=a \varphi
$$

Finally we obtain:

$$
d s^{2}=-d t^{2}+d r^{2}+r^{2} d \tilde{\varphi}^{2}+d z^{2}
$$

Which obviously is flat space (locally)in cylindrical Weyl coordinates. We mention here that the flat space obtained here has a conical nature. As we noted in [14] the conical parameter $a$ related to the gravitational mass per unit length of the spacetime, $\eta$, as

$$
a=1-4 \eta
$$

Such that $0<a<1$. This metric, exposing the geometry around a straight cosmic string, which is locally identical flat spacetime however it is not globally Euclidian, since the angle $\tilde{\varphi}=a \varphi$ varies in the range $0<\tilde{\varphi}<B$ where $b=2 \pi a=2 \pi(1-4 \eta)$.

\section{Case (2) : solution with $u(r)=v(r)=w(r) \neq$ constant, $\phi^{\prime} \neq 0$}

We must mention that the system of differential equations $(3,4)$ posses a special solution $\left\{u_{i}(r)\right\}=u(r)$ for the following equations:

$$
\begin{array}{r}
2 u^{\prime \prime}+3 u^{\prime 2}-4 \Lambda=0 \\
6 u^{\prime \prime}+3 u^{\prime 2}-4 \Lambda=4 \phi^{\prime 2}
\end{array}
$$

Immediately we can obtain an integral for the field:

$$
\phi= \pm \int \sqrt{u^{\prime \prime}} d r
$$

The differential equation (5) is a non linear 2'nd order,and posses the general solution for metric function $u(r)$ :

$$
u(r)= \pm 2 \sqrt{\frac{\Lambda}{3}} r+\frac{1}{3} \log \left[\frac{3}{16 \Lambda}\left(c_{1} e^{2 \sqrt{3 \Lambda} r}-c_{2}\right)^{2}\right]
$$

The differential equation (5) is solved by taking $u(r)=a r+y(r)$, matching constant value $a$ and solving differential equation $2 y^{\prime \prime}+y^{\prime 2}-4 \Lambda y^{2}=0$ or by a simple change of variable from $u(r)$ to $p=u^{\prime}$ and consequently rewriting (5) to a first order differential equation for $p(u)$.

Substituting (8) in (7) we obtain:

$$
\phi(r)=\mp 2 i \frac{\sqrt{6}}{3} \tanh ^{-1}\left(\sqrt{\frac{c_{1}}{c_{2}}} e^{\sqrt{3 \Lambda} r}\right)
$$


Substituting (8) in (1) and calculating Ricci scalar we obtain:

$$
R=4 \Lambda\left[\frac{c_{2}^{2}+c_{1}^{2} e^{4 \sqrt{3 \Lambda} r}-4 c_{1} c_{2} e^{2 \sqrt{3 \Lambda} r}}{\left(c_{1} e^{2 \sqrt{3 \Lambda} r}-c_{2}\right)^{2}}\right]
$$

Contraction the field equation (2) and substituting metric function $u$, with the field solution gives the same result. Scalar field must be a real function, then it is proper to define a new parameter as:

$$
\sqrt{\frac{c_{1}}{c_{2}}}=i \xi
$$

Which in term of $\xi$ the metric and field functions $(8,9)$ transform to functions:

$$
\begin{array}{r}
u(r)= \pm 2 \sqrt{\frac{\Lambda}{3}} r+\frac{1}{3} \log \left[\left(\xi^{2} e^{2 \sqrt{3 \Lambda} r}+1\right)^{2}\right] \\
\phi= \pm 2 \frac{\sqrt{6}}{3} \tan ^{-1}\left(\xi e^{\sqrt{3 \Lambda} r}\right)
\end{array}
$$

We attend that only the minus sign in metric function is consistent with scalar field equation of motion $\square \phi=0$ and the positive signs makes it with wrong conclusion ${ }^{3}$. Remind that we take $c_{2}=4 \sqrt{\frac{\Lambda}{3}}$ to eliminate the constant term. Also Ricci scalar (10) can be written as:

$$
R=4 \Lambda\left[\frac{1+\xi^{4} e^{4 \sqrt{3 \Lambda} r}+4 \xi^{2} e^{2 \sqrt{3 \Lambda} r}}{\left(\xi^{2} e^{2 \sqrt{3 \Lambda} r}+1\right)^{2}}\right]
$$

And the final form of metric (1) using equation (11) is:

$$
d s^{2}=d r^{2}+e^{-2 \sqrt{\frac{\Lambda}{3}} r}\left(\xi^{2} e^{2 \sqrt{3 \Lambda} r}+1\right)^{2 / 3}\left(-d t^{2}+d \varphi^{2}+d z^{2}\right)
$$

This is the main result of our article. We obtained an exact solution which contains two parameter: Cosmological constant term $\Lambda$ and a parameter $\xi$ associated directly to the scalar field. Indeed the constant $\xi$ determines how strongly the scalar field creates curvature.Clearly the above metric permits 1 time-like Killing fields $\frac{\partial}{\partial t}$, which denotes that the present solution is static, and 3 space-like Killing fields (including 2 translational fields and 1 rotational ones), which span a Euclidean group $G(3)$. The cosmological horizon(s) for cylindricall symmetric spacetimes is discussed completely by Wang [18]. The no singularity structure of the solution (14) is apparent from its Kretschmann scalar.It reads

$$
R_{\mu \nu \alpha \beta} R^{\mu \nu \alpha \beta}=\frac{3}{8}\left[\left(\frac{f^{\prime}}{f}\right)^{4}+2\left(\frac{f^{\prime \prime}}{f}\right)^{2}-2\left(\frac{f^{\prime}}{f}\right)^{2} \frac{f^{\prime \prime}}{f}\right]
$$

Where we define in:

$$
f=e^{-2 \sqrt{\frac{\Lambda}{3}} r}\left(\xi^{2} e^{2 \sqrt{3 \Lambda} r}+1\right)^{2 / 3}
$$

\footnotetext{
${ }^{3}$ If one write the equation of motion for a massless minimally coupled scalar field in a metric like (14) $d s^{2}=$ $d r^{2}+f(r)\left(-d t^{2}+d \varphi^{2}+d z^{2}\right)$ we observe that $f^{3 / 2}(r) \phi^{\prime}=$ c.t.e. By writing $f(r)=e^{ \pm 2 \sqrt{\frac{\Lambda}{3}} r}\left(\xi^{2} e^{2 \sqrt{3 \Lambda} r}+1\right)^{2 / 3}$ and $\phi= \pm 2 \frac{\sqrt{6}}{3} \tan ^{-1}\left(\xi e^{\sqrt{3 \Lambda} r}\right)$ obviously this statement is proved.
} 
The poles of Kretschmann in real $r$ plane is determined by roots of equation $f=0$. So we can conclude the following results

1-If $\xi^{2}>0$ i.e. the scalar field's parameter is a real constant, Then the Kretschmann scalar has no naked singularity at $r=r_{0}$.

2- If we leave the above condition and allow $\xi$ to be a complex value then the exact solution (14) has naked singularity at $r=r_{0}=-\frac{1}{3} a \ln (|\xi|)$ where $|\xi|=\operatorname{Modulus}(\xi)$. Only for $0<|\xi|<1$ this naked singularity is physically plausible. If $|\xi|>1$ the exact solution has no naked singularity. Thus for our next cosmological discussions that it is limited to $\xi^{2}>0$ and real scalar fields, we can tell that our solution has no singularity.

\section{RECOVERING PREVIOUS SOLUTIONS}

In this section we show that our solution gives $L C \Lambda$ and Buchdahl solutions.

\section{Case (1) : limit of the solution with $\Lambda \neq 0, \phi=$ constant ( $L C \Lambda$ family)}

We note that if $\xi \rightarrow 0$ the scalar field disappears and the Ricci scalar (13) becomes $4 \Lambda$ which is trivial in vaccum limit of relation $(2)^{4}$. The line element $(1)$ in this case is read as:

$$
d s^{2}=d r^{2}+e^{ \pm 2 \sqrt{\frac{\Lambda}{3}} r}\left(-d t^{2}+d \varphi^{2}+d z^{2}\right)
$$

This space time is the other solution of General $L C \Lambda$ family which has studied in [5]. Because $r=0$ represents no special hypersurface, we can also extend the range of $r$ to $(-\infty, \infty)$ and write the metric with the + sign only.Spacetime with metric (15)constitutes part of deSitter spacetime with metric written in the horospherical coordinates. Solution (14) with the + sign covers part of de -Sitter hyperboloid with $x \in\left(0, \sqrt{\frac{3}{\Lambda}}\right]$ while the - sign solution is valid for $x \in\left[\sqrt{\frac{3}{\Lambda}}, 0\right)$ which $r= \pm \sqrt{\frac{3}{\Lambda}} \log \left(x \sqrt{\frac{\Lambda}{3}}\right)$ in .

\section{Case (2) : limit of the solution with $\Lambda=0, \phi \neq \operatorname{constant(Buchdahl~family)~}$}

If $\Lambda \rightarrow 0$ we must at least recover one member of Buchdahl cylindrical family. First we remind that from Buchdahl theorem the general form of a massless minimally coupled scalar field in axial symmetry has the following metric form ${ }^{5}$ :

$$
d s^{2}=e^{2(\gamma-\beta \psi)}\left(d \rho^{2}+d z^{2}\right)+\rho^{2} e^{-2 \beta \psi} d \varphi^{2}-e^{2 \beta \psi} d t^{2}
$$

In which the metric functions $\psi, \gamma$ satisfy the following equations:

$$
\begin{array}{r}
\rho^{-1}\left(\rho \psi_{, \rho}\right)_{, \rho}+\psi_{, z z}=0 \\
\gamma(\rho, z)=\int\left[\left(\rho \psi_{, \rho}^{2}-\psi_{, z}^{2}\right) d \rho+2 \rho \psi_{, \rho} \psi_{, z} d z\right] \\
\phi=2 \lambda \psi \\
\beta= \pm\left(1-2 \lambda^{2}\right)^{1 / 2}
\end{array}
$$

\footnotetext{
${ }^{4}$ remind that this case trivially $\square \phi=0$ satisfied and both signs \pm in metric is admittable

${ }^{5}$ Conventions on metric follow Buchdahl[1]
} 
If $\beta=1$ or $\lambda=0$ we obtain the seed vacuum solution in axial symmetry, and if we focused only on cylindrical one, we would have Levi-Civita solution [8]. We must show that the metric arisen from function (11) in limit $\Lambda \rightarrow 0$ belongs to this family. The limit $\Lambda \rightarrow 0$ of the field (12) must coincide with Buchdahl S-field for some value of $\lambda, \psi$. Now we re derive the general form of Buchdahl metric in cylindrical symmetry. If the metric functions (16) depend only on radial coordinate $\rho$,from equations $(17,18)$ we obtain:

$$
\begin{aligned}
& \gamma(\rho)=c_{1}^{2} \log (\rho)+c_{3} \\
& \psi(\rho)=c_{1} \log (\rho)+c_{2}
\end{aligned}
$$

After substituting these functions in (16) and comparing with metric mass parameter, $m$,of Levi-Civita solution, also rewriting with the non radial coordinates $(t, \varphi, z) \rightarrow(\tilde{t}, \tilde{\varphi}, \tilde{z})$ we finally have:

$$
d s^{2}=\rho^{2 m(m-\beta)}\left(d \rho^{2}+d \tilde{z}^{2}\right)+\rho^{2-2 \beta m} d \tilde{\varphi}^{2}-\rho^{2 \beta m} d \tilde{t}^{2}
$$

Since we work on the gauge (1), it is suitable to convert Buchdahl metric (21) to our gauge. So we apply the radial coordinate transformation :

$$
\rho=\left[\left(m^{2}-m \beta+1\right) r\right]^{\frac{1}{m^{2}-m \beta+1}}
$$

In term of this coordinate ,metric (21) transforms to:

$$
d s^{2}=d r^{2}-g_{t t} d t^{2}+g_{\varphi \varphi} d \varphi^{2}+g_{z z} d z^{2}
$$

Which we have:

$$
\begin{aligned}
g_{t t} & =\left[\left(m^{2}-m \beta+1\right) r\right]^{\frac{2 m \beta}{m^{2}-m \beta+1}} \\
g_{\varphi \varphi} & =\left[\left(m^{2}-m \beta+1\right) r\right]^{\frac{2-2 m \beta}{m^{2}-m \beta+1}} \\
g_{z z} & =\left[\left(m^{2}-m \beta+1\right) r\right]^{\frac{2 m(m-\beta)}{m^{2}-m \beta+1}}
\end{aligned}
$$

Now we write the line element for $(1)$ in limit $\Lambda \rightarrow 0$.Using field equations $(5,6)$ in this limit and supposing $\phi(r) \neq$ c.t.e (which leads to flat space as it is shown previously) we can write the line element:

$$
d s^{2}=d r^{2}+\left[\frac{3}{2}\left(c_{1} r+c_{2}\right)\right]^{\frac{2}{3}}\left(-d t^{2}+d \varphi^{2}+d z^{2}\right)
$$

Without loss of generality we can take $c_{2}=0$. Comparing metric (22) with (23), term by term, we obtain the following simple algebraic equations:

$$
\begin{aligned}
\frac{3}{2} c_{1}=m^{2}-m \beta & +1 \\
m \beta & =1 \\
m^{2}-4 m \beta+1 & =0
\end{aligned}
$$

The solutions of these equations are:

$$
\begin{array}{r}
m= \pm \sqrt{3} \\
\beta= \pm \frac{\sqrt{3}}{3} \\
c_{1}=2
\end{array}
$$


By substituting of $\beta$ in (20) we have $\lambda= \pm \frac{\sqrt{3}}{3}$. Thus we show that our exact solution gives at least one Buchdahl solution with proper values of $\beta, m$. We note here that only in vaccum case $m= \pm 1$ it corresponds to flat space[7] and in non vaccum case (as Buchdahl family which contains Scalar field) it is a non flat spacetime. Also if one can solve $(3,4)$ completely we expect that the solution recovers all Buchdahl family with desired values of $\beta, m$.

\section{COSMOLOGICAL SOLUTIONS}

As we know, in General relativity, It is conceivable that some of the solutions are related to cosmological solutions through a time-dependent scalar field with the cosmological constant.For an example we can treat a massless minimally coupled scalar field in spherically symmetric space time as a perfect fluid with equation of state $p=-\rho$ where $p$ is the principal pressure (radial pressure in spherical symmetry) and $\rho$ the energy density of scalar field enclosed spacetime [16]. As i mentioned in [3] this is a triviality in spherical symmetry.There is no simple physical mechanism hidden behind it. Many exact solutions have found describe perfect fluids with a general equation of state $p=f(\rho)$. Taub and Tabensky show that in plane symmetry, a perfect fluid solution can admit a scalar field stress-energies [12]. Our solutions which is derived in (8) can be converted to time-dependent metrics via the complex transformation $r \rightarrow i \tau$ and $t \rightarrow i u$, as Vaidya and Som discussed in[13]. Since the scale factor of the resulting metric should be a real one valued function of time like coordinate $w$, the physical, well defined and meaningful solution is obtained by choosing the first linear solution of (5).As we showed in case (1) this solution is a member of $L C \Lambda$ family.Another Cosmological solution can be obtained by choosing a complex transformation and having $\xi^{2}=1$. This cosmology describe as Case (2).

Case (1) : Cosmological model $\Lambda<0, \xi^{2}=0$

Now we prove that under complex transformation this metric describe static anti de Sitter space universe with a constant Hubble parameter $H=\sqrt{\frac{-\Lambda}{3}}$. This case is occurred for a negative Cosmological constant. Albeit this solution is in contradiction to our intuition about the recent epoch of universe which persuaded physicists to accept a positive cosmological constant, theoretically it's importance in relation to AdS correspondence and String Theory is momentous. If we apply $r \rightarrow i \tau$ and $t \rightarrow i u$ in - sign solution of (15) we obtain:

$$
d s^{2}=-d \tau^{2}+e^{2 \sqrt{\frac{-\Lambda}{3}} \tau}\left(d u^{2}+d \varphi^{2}+d z^{2}\right)
$$

The line element (24) is correspond to a Friedmann Cosmology with Hubble parameter

$H=\sqrt{\frac{-\Lambda}{3}}$ in the coordinates $(\tau, u, \varphi, z)$. We can interpreted this solution as an inflationary model where the inflaton, whose energy density is roughly constant, dominates the cosmic evolution and determines the (roughly constant) Hubble parameter while causing the scale factor to grow exponentially. 
Case (2) : Cosmological model $\Lambda>0, \xi^{2}=1$

The metric function in (14) must be a real function even in analytic continuation of $t, r$ (complexified $t, r$ ).We observe that if we choose the minus sign solution and set $\xi^{2}=1$ in (14) the metric can then be converted to RobertsonWalker form:

$$
d s^{2}=-d \tau^{2}+\left(2 \cos \left(\frac{3 \tau}{a}\right)\right)^{2 / 3}\left(d u^{2}+d \varphi^{2}+d z^{2}\right)
$$

Where we define a de-Sitter radius as $a=\sqrt{\frac{3}{\Lambda}}$. This form of solution suggests time coordinate $\tau$ as a periodic coordinate.In the case of Cylindrical topology of the spatial sections, $\tau, z$ and $\varphi$ are interpreted as generalized Euler angle coordinates. Cyclic cosmology are based on the braneworld picture of the universe [15], in where spacetime is effectively 5-dimensional,with one dimension which is not extending indefinitely, but being a line segment. The cyclic model is an ambitious attempt to provide a complete history of the universe, within the framework of a braneworld view of the universe, while incorporating both the ekpyrotic mechanism and dark energy in an essential way. This solution repeated every once by a period $T=\frac{2 \pi a}{3}$. The metric (25) as a Freidmann model has a bounded scale factor, i.e. for all epoches of the universe it satisfies the following inequality(in natural units),

$$
a(\tau) \leq 1.2599
$$

\section{SUMMARY AND CONCLUSIONS}

Exact solution of Einstein theory is discussed in many different Symmetries with a diversified class of stress-energies. For a scalar field there is only a few exact solutions in spherical, cylindrical or plane symmetries. In this manner the generating methods are so important. one of the simple ones which is applicable for a massless scalar field is Buchdahl Reciprocal method that if we applied it to a static vaccum solution of Einstein equations in cylindrical symmetry we would obtain a one parameter exact solution which recovers seed metric (LC spacetime) in a special case. In this article we generalized Buchdahl metric to that one with a cosmological constant term and by considering more general cylindrical metric in Weyl gauge, we found a new 2 parameter exact solution for a massless scalar field in the presence of the cosmological constant term. Far from the importance of this solution as a new exact solution, this solution can be interpreted as a cosmological solution by complexities radial and time coordinates. Two distinct classes of solutions to the problem of a cylindrically-symmetric static field in general relativity with cosmological constant have been found. The first one of these solutions involves two parameters, cosmological constant term $\Lambda$ and $\xi$, which is related to a scalar field. Various choices of $\xi$ demonstrated that the presented solution was a generalization of the metrics previously derived by Buchdahl, Linet [10] and Tian [11]. These solutions are static Cosmic string spacetimes. Another solution contains flat space. The limit of solution in the absence of scalar field coincides with a number of $L C \Lambda$ family which has many attractive properties . As an example, Spacetime with this metric constitutes part of de- Sitter spacetime with metric which is written in the horospherical coordinates. We also present two cosmological models by analytic continuation the time and radial coordinates. First one corresponds to an inflationary model that 
the Hubble parameter remains constant in it and has no dynamics. Another Cosmological solution is a cosmic time periodic model which can be classified as a Cyclic model which has some important applications in braneworld scenario.

\section{ACKNOWLEDGEMENT}

D.Momeni thanks Anzhong Wang for useful comments and valuable suggestions.

$\dagger$ Electronic address: davood.momeni@kiau.ac.ir

‡ Electronic address: miraghaee @ physics.sharif.ir

[1] H. A. Buchdahl. Phys. Rev. 115, 1325-1328 (1959)

[2] M. Wyman. Phys. Rev. D. vol 40, Num 8 (1981)

[3] D. Momeni. arXive:gr-qc/0903-0067 (2009)

[4] B. C. Xanthopolous and T. Zannias. Phys. Rev. D. vol 40, Num 8 (1989)

[5] M. Zofka, J. Bicak. Class.Quant.Grav.25:015011,(2008)

[6] C. Vuille. Gen Relativ Gravit (2007) 39:621632 (2007)

[7] H. Stephani, D. Kramer, M.A.H. MacCallum, C. Hoenselaers and E. Herlt, Exact solutions of Einsteins field equations, 2nd ed. (Cambridge: Cambridge University Press) (2003).

[8] T. Levi-Civita, Rend. Acc. Lincei 27 (1917) 183.

[9] A. Z. Wang. Phys. Rev. D.68 (2003) 064006

[10] B. Linet, J. Math. Phys. 27 (1986) 1817.

[11] Q. Tian, Phys. Rev. D 33 (1986) 3549.

[12] Tabensky, R.R., Taub, A.H.: Commun. Math. Phys. 29, 61 (1973)

[13] Vaidya, A.M., Som, M.M.: Phys. Rev. D 27(8), 17281730 (1983)

[14] A. Azadi , D. Momeni , M. Nouri-Zonoz: Physics Letters B 670 (2008) 210214

[15] J. L. Lehners, P. Smyth and K. S. Stelle, Nucl. Phys. B 790, 89 (2008) arXiv:0704.3343 [hep-th]]

[16] D. Momeni,A. Azadi.: Astrophysics and Space Science, vol. 317, no. 3.(2008)-pp. 231-234

[17] O. Sarioglu, B. Tekin , arXiv:0901.1242 [gr-qc] ( To appear in Phys Rev D)

[18] Anzhong Wang : Phys.Rev. D72 (2005) 108501 